\title{
Working with patients to improve care
}

\author{
Tara Kiran MD MSc, Joshua Tepper MD MPH MBA, Frank Gavin MA
}

Cite as: CMAJ 2020 February 10;192:E125-7. doi: 10.1503/cmaj.190439

$\mathrm{H}$ ealth care organizations exist to provide care to patients. Yet recent public debates on hospital parking and access to health records show that, far too often, decisions about how health services are designed and delivered have been made by managers and clinicians alone. Health care professionals are taught to be patient centred in the context of a clinical encounter, but rarely are clinicians taught to be patient centred when it comes to how they run their clinics, conduct ward rounds or schedule surgeries. Designing a health care system that puts patients at the centre requires their engagement. Patient engagement means patients and health care professionals working in active partnership at all levels - direct care, organizational design and governance, and policy-making - to improve health and health care. ${ }^{1}$ This means engaging patients to improve quality within health care organizations, including hospitals, primary care clinics and long-term care homes. We discuss strategies for engagement, address common challenges and reflect on next steps. We use the term "patients" for brevity, but mean it to include family and caregivers.

Engaging patients in health service design and delivery can improve care. A 2017 systematic review found few robust studies evaluating the impact of patient advisers on health outcomes but noted case studies that described improvements in appointment access, patient safety and patient satisfaction. ${ }^{2} \mathrm{~A}$ more recent systematic review found that patient engagement resulted in enhanced care or service delivery, particularly if an organization used methods of codesign. ${ }^{3}$ Experience-based codesign begins with an in-depth exploration of the experiences and emotions of patients and then brings patients and staff together to identify and implement improvements in service. ${ }^{4}$ Numerous case examples illustrate the impact of codesign on organizational service delivery, including in our own work, where it led to improved patient-reported after-hours access in primary care. ${ }^{5}$

Moreover, patient engagement leads to new and important insights. For example, patient involvement in reporting of safety incidents highlighted safety concerns that organizations' traditional safety reporting systems overlooked. ${ }^{6}$ Patients in our practice helped us reframe our own concepts of timely access in primary care and how we measure it. ${ }^{5}$ Crucially, patient engagement can help us develop and test ideas for improving care. For example, we recently tried to improve cancer screening rates for patients living with a low income. We initially imagined that the intervention

\section{KEY POINTS}

- Engaging patients in health service design and delivery can improve care, lead to new and important insights, and be a positive experience for both patients and providers.

- Engaging patients in service improvement is new for many physicians and managers, but resources exist to help them get started.

- It is important to clarify the purpose of the engagement, recruit and support a diverse mix of patients and consider compensating them for their time and contributions.

- For patient engagement to be meaningful, organizations need to commit to acting on at least some of the recommendations arising from the engagement, and should transparently report on progress and/or reasons why recommendations could not be acted on.

would involve a low-literacy mailed handout, but patients told us they often did not open their mail and instead wanted a convivial group education session. ${ }^{7}$

Patient engagement can be a positive experience for both patients and providers. Engaging patients in clinic design and program delivery is a natural extension of the concept of patientcentredness that we value in individual clinician-patient interactions and can help ground clinicians in their professional purpose. It also aligns with our democratic ideals that value the experience and contribution of all citizens to creating a better society. In our experience, patients convey not just where care can be improved but also what is already working well; the latter can be heartening for staff. Patients often volunteer as a way, they say, to give back to their community. Their altruism can be fortifying, even inspiring. In some cases, patients who are engaged have improved outcomes themselves. ${ }^{2}$

Even though engaging patients to improve services is beneficial, it is also new for many clinicians and managers. Figure 1 offers suggestions for getting started by listing a range of methods that require varying levels of resources from providers and patients. Methods are presented alongside a continuum of engagement as outlined in the Patient Partnering Framework developed by Health Quality Ontario. ${ }^{8}$ Although deeper engagement (such as patient partnership) has the potential for the most profound and meaningful impact, ${ }^{3}$ other levels of engagement (such as consulting patients) should not necessarily be dismissed 


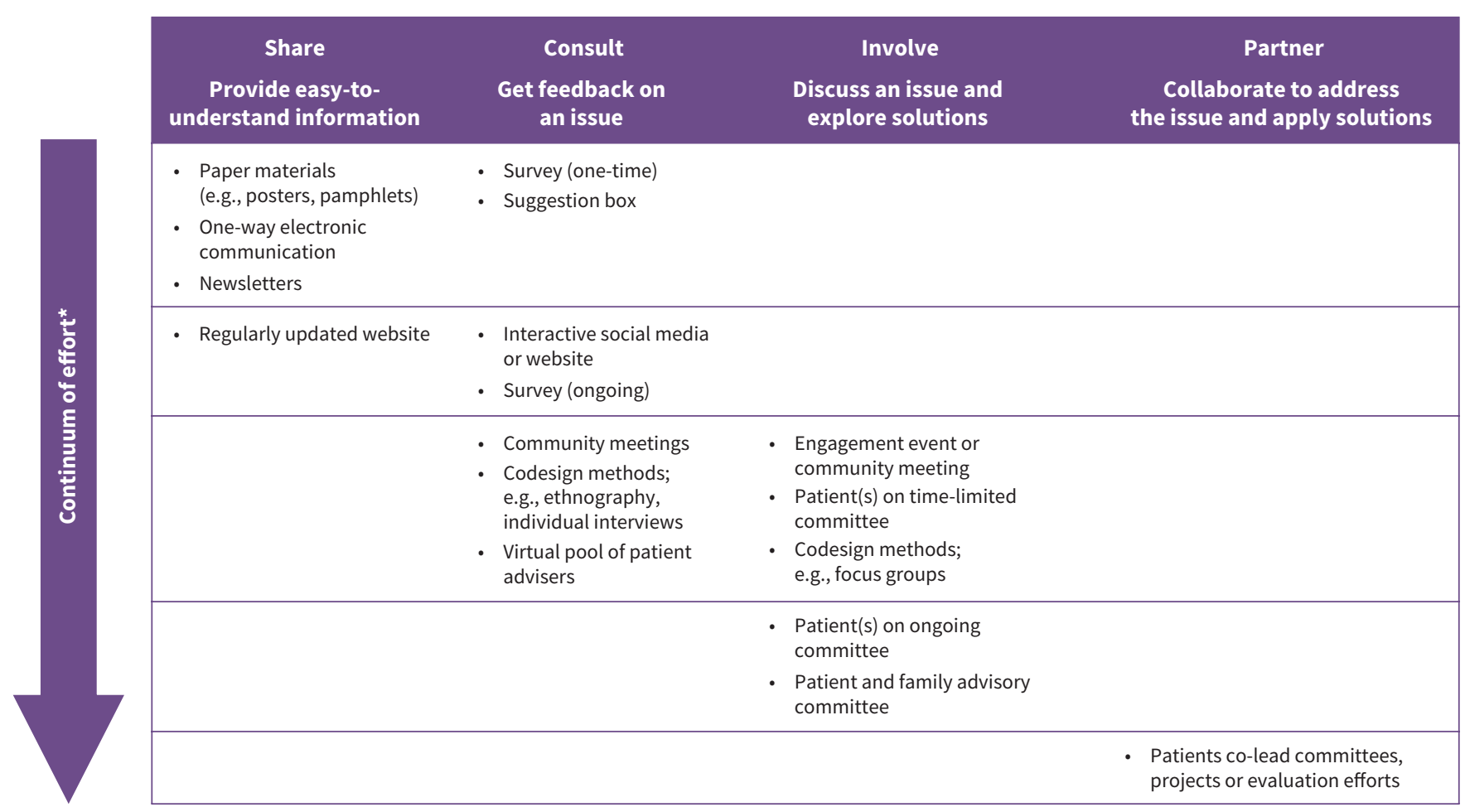

Figure 1: Methods for engaging patients. Note: *Generally requires more resources, provider skill and patient commitment.

as tokenistic. They may be all that is possible, especially for some patients, and in many cases, they may be all that is needed.

Regardless of the method and level of patient engagement, engaging patients in service improvement requires careful planning and execution, key considerations of which are outlined in Box 1. To start, organizations should clarify the purpose of the engagement - preferably, together with patients - and choose methods accordingly. Recruiting patients from diverse backgrounds requires thoughtful outreach and scheduling, a range of engagement methods, efforts to accommodate unique needs, and data collection to understand sociodemographic diversity. Patients should be encouraged not to think of themselves as representing a particular group or viewpoint, but to draw upon their own experience while also considering the needs of others.

Sociodemographic diversity helps ensure that perspectives and solutions reflect differences in lived experience - differences that are sometimes profound, that ideally lead to respectful discussion and that sometimes identify common needs. Adapting the use of civic lotteries in health care is one promising approach to supporting diverse engagement. ${ }^{5}$ Deliberate inclusion of diverse voices in patient engagement can also be achieved by drawing on published best practices. ${ }^{9}$ Compensation of patient partners can help increase diversity but can be tricky for small organizations with limited budgets. Existing frameworks, such as the Change Foundation's tool for deciding whether to pay patient-engagement participants, can provide useful guidance. ${ }^{10}$

\section{Box 1: Considerations when engaging patients: CARE2}

- Clarify: Work with patients to clarify the purpose of the engagement, the process and anticipated outcomes.

- Accommodate: Support patients from a variety of backgrounds to participate, by meeting unique needs related to, for example, scheduling, childcare, transportation, language and disability.

- Reach out: Seek diverse voices through thoughtful recruitment. Collect demographic information of participants to guide recruitment efforts.

- Educate each other: Share your "insider knowledge" of the health care system and provide opportunities for patients to share their "insider knowledge" of living with a condition.

- Compensate: Decide how to recognize and compensate patients for their time and contribution. Providing food and transportation is a basic requirement.

- Act: Identify how you will act on the input, ensure followthrough and communicate the results in a timely way.

- VaRy it up: Consider trying a variety of engagement methods and engaging at varying points in your improvement journey.

- Evaluate: Elicit and respond to feedback from patients about the engagement process.

To participate in tackling complex issues, patients may initially need education about the health system to problem-solve effectively. Likewise, health care professionals often need guidance on how to work successfully with patients. Organizations can use 
icebreakers, storytelling (by patients and providers), small group interactions and explicit reframing of the patient and provider roles to help equalize traditional health care hierarchies. ${ }^{11}$

For patient engagement to be meaningful, organizations need to commit to acting on at least some of the recommendations arising from the engagement and should report transparently on progress, however much or little there has been. When an organization is not able to implement a recommendation, patients may find it reassuring when the response makes clear that they were heard, and their recommendation was carefully considered.

Several quality and research agencies in Canada have guidance to support patients and health care organizations to work together to improve care. We can also learn from the experiences of other jurisdictions. In 2011, the United Kingdom introduced a financial incentive for primary care practices to set up "patient participation groups." 12 However, early evaluations suggested that general practitioners were initially suspicious of efforts, sometimes perceiving a threat to their autonomy. Patient participants were often not representative of the practice, in terms of age and ethnicity, and often participants felt their voices were not heard. Research suggested that the groups would benefit from clearer goals, resources, training and support. ${ }^{12}$ These experiences are particularly relevant given that patient and family advisory committees are the most common way that organizations engage patients in Canada currently.

It is crucial that health care organizations can commit dedicated resources both to facilitate the effective engagement of patients using a broad range of methods and to build related expertise. Smaller organizations may need to partner with others to work with patients in a meaningful and inclusive way. Rather than mandating patient engagement, which may lead to tokenism, we should work to shift medical cultural norms, starting with explicitly teaching medical learners about the importance of engaging patients and how to do it. Finally, we need more rigorous and systematic evaluation of the impact of patient engagement on health outcomes; of how context and mechanisms influence the impact; and of system costs, including potential harms.
Meaningful partnership with patients to improve the design and delivery of services can reorient the system to those it is meant to serve. Don Berwick once said that clinicians are guests in patients' lives. ${ }^{13}$ As good guests do, clinicians should follow the lead of our hosts and allow them to arrange the venue, set the menu and guide the conversation.

\section{References}

1. Carman KL, Dardess $P$, Maurer M, et al. Patient and family engagement: a framework for understanding the elements and developing interventions and policies. Health Aff (Millwood) 2013;32:223-31.

2. Sharma AE, Knox M, Mleczko VL, et al. The impact of patient advisors on healthcare outcomes: a systematic review [published erratum in BMC Health Serv Res 2018;18:437]. BMC Health Serv Res 2017;17:693.

3. Bombard Y, Baker GR, Orlando E, et al. Engaging patients to improve quality of care: a systematic review. Implement Sci 2018;13:98.

4. EBCD: experience-based co-design toolkit. London (UK): The Point of Care Foundation. Available: www.pointofcarefoundation.org.uk/resource/experience -based-co-design-ebcd-toolkit/ (accessed 2019 Sept. 30).

5. Kiran T, Ramji N, Derocher MB, et al. Ten tips for advancing a culture of improvement in primary care. BMJ Qual Saf 2019;28:582-7.

6. O'Hara JK, Reynolds C, Moore S, et al. What can patients tell us about the quality and safety of hospital care? Findings from a UK multicentre survey study. BMJ Qual Saf 2018;27:673-82.

7. Lofters AK, Baker NA, Schuler A, et al. A "tea and cookies" approach: co-designing cancer screening interventions with patients living with low income. J Gen Intern Med 2019 Oct. 21 [Epub ahead of print]. doi: 10.1007/s11606-019-05400-0.

8. Patient Partnering Framework. Health Quality Ontario. Available: www.hqontario. ca/Patient-Partnering/Patient-Partnering-Framework (accessed 2019 Apr. 16).

9. Snow B, Tweedie K, Pederson A, et al.; AWESOME Project Team. Patient engagement: heard and valued - a workbook for meaningful engagement of patients that have not traditionally been heard in healthcare planning. Canadian Foundation for Healthcare Improvement; 2013. Available: www.cfhi-fcass. ca/sf-docs/default-source/patient-engagement/awesome_workbook-fraserhealth .pdf (accessed 2019 Sept. 30).

10. Should money come into it? A tool for deciding whether to pay patientengagement participant. Toronto: The Change Foundation. Available: www. changefoundation.ca/patient-compensation-report/ (accessed 2019 Apr. 16).

11. Dainty K, Kiran T. "Spending the day with your Family Health Team": rapid ethnography of a patient-centred quality improvement event. BJGP Open. doi: https://doi.org/10.3399/bjgpopen20X101002.

12. Gillam S, Newbould J. Patient participation groups in general practice: what are they for, where are they going? BMJ 2016;352:i673.

13. Berwick DM. The epitaph of profession. Br J Gen Pract 2009;59:128-31.

\begin{abstract}
Competing interests: Tara Kiran is the Fidani Chair of Improvement and Innovation at the University of Toronto. She is supported as a Clinician Investigator by the Departments of Family and Community Medicine at St. Michael's Hospital and the University of Toronto. She also receives support from the Canadian Institutes of Health Research and Ontario Health's Quality Business Unit (formerly Health Quality Ontario) in her role as an embedded clinician researcher. No other competing interests were declared.
\end{abstract}

This article was solicited and has been peer reviewed.
Affiliations: Department of Family and Community Medicine (Kiran, Tepper), St. Michael's Hospital; Department of Family and Community Medicine (Kiran, Tepper), University of Toronto; MAP Centre for Urban Health Solutions (Kiran), Li Ka Shing Knowledge Institute, St. Michael's Hospital, Toronto, Ont.; North York General Hospital (Tepper), North York, Ont.; Institute of Health Policy, Management, and Evaluation (Kiran, Tepper), University of Toronto; Ontario Health, Quality Business Unit (Kiran), Toronto, Ont.; CHILD-BRIGHT Network (Gavin), Montréal, Que.; Ontario Strategy for Patient-Oriented Research Support Unit (Gavin), Toronto, Ont.
Contributors: All of the authors contributed to the conception and design of the work. Tara Kiran drafted the manuscript. All authors revised it critically for important intellectual content, gave final approval of the version to be published and agreed to be accountable for all aspects of the work.

Acknowledgements: The authors thank Marwan Asalya and Jennifer Quaglietta from North York General Hospital for providing ideas for the figure summarizing methods of engagement.

Correspondence to: Tara Kiran, tara.kiran@utoronto.ca 